ESAIM: PROCEEDINGS, March 2012, Vol. 35, p. 203-209

Fédération Denis Poisson (Orléans-Tours) et E. Trélat (UPMC), Editors

\title{
AN ANTI-DIFFUSIVE LAGRANGE-REMAP SCHEME FOR MULTI-MATERIAL COMPRESSIBLE FLOWS WITH AN ARBITRARY NUMBER OF COMPONENTS
}

\author{
Marie Billaud Friess ${ }^{1}$ and Samuel KoKh ${ }^{2}$
}

\begin{abstract}
We propose a method dedicated to the simulation of interface flows involving an arbitrary number $m$ of compressible components. Our task is two-fold: we first introduce a $m$-component flow model that generalizes the two-material five-equation model of $[2,3]$. Then, we present a discretization strategy by means of a Lagrange-Remap $[8,10]$ approach following the lines of $[5,7,12]$. The projection step involves an anti-dissipative mechanism derived from $[11,12]$. This feature allows to prevent the numerical diffusion of the material interfaces. We present two-dimensional simulation results of threematerial flow.

Résumé. Nous proposons une méthode de simulation pour des écoulements comportant un nombre arbitraire $m$ de composants compressibles séparés par des interfaces. Nous procédons en deux étapes : tout d'abord nous introduisons un modèle d'écoulement à $m$ composants qui généralise le modèle à cinq équations de $[2,3]$. Ensuite nous présentons une stratégie de discrétisation de type LagrangeProjection $[8,10]$ inspirée de $[5,7,12]$. La phase de projection met en ouvre une technique de transport anti-diffusive $[11,12]$ qui permet de limiter la diffusion numérique des interfaces matérielles. Nous présentons des résultats de calcul bidimensionnel d'écoulement à trois composants.
\end{abstract}

\section{INTRODUCTION}

In this paper, we are interested in the simulation of a multicomponent flows with sharp interfaces that separate $m$ distinct materials on a Cartesian grid. Each material is supposed to be a compressible fluid equipped with its own Equation of State (EOS) and all viscosity effects are neglected. We consider an approach that relies on $m$ variables $\mathcal{Z}_{k}, k=1, \ldots, m$ usually called colour functions. The function $\mathcal{Z}_{k}$ take the value 1 in pure fluid $k$ regions and 0 elsewhere. This method allows to choose the relevant EOS at each point of the domain. In this context the material interfaces are discontinuity locus of the colour functions. The interfaces motion is governed by imposing each $\mathcal{Z}_{k}$ to verify a transport equation.

With a focus on ease of implementation, we take advantage of this Eulerian approach by choosing Eulerian Finite-Volume discretization strategies for all the variables. The update of the interfaces position is obtained by approximating the evolution equation of each $\mathcal{Z}_{k}$. As a result, for standard numerical schemes, the interfaces are numerically spread over a few grid cells by common numerical methods and the sharp interface model is no longer valid. A classical cure consists in introducing a numerical transition model that allows the interface to

\footnotetext{
1 LUNAM Université, GeM UMR CNRS 6183, Ecole Centrale Nantes, Université de Nantes

1 rue de la Noë, BP 92101, 44321 Nantes Cedex 3 (France); e-mail: marie.billaud-friess@ec-nantes.fr

2 DEN/DANS/DM2S/STMF/LMEC , CEA Saclay, 91191 Gif-sur-Yvette (France); e-mail: samuel.kokh@cea.fr
}

(C) EDP Sciences, SMAI 2012 
be diffused while converging towards the right discontinuity when the discretization space step tends to zero. To this purpose we adopt an extend for an arbitrary $m \geq 2$ the model proposed in $[2,3]$ that deals with the case $m=2$. The model we propose here is hyperbolic under simple hypotheses pertaining to the pure fluid EOS's.

Although the above approach succeeds in simulating $m$-component interface flows, the structure of the interface may be altered by numerical diffusion throughout the computation. Our second task is to adress this issue by designing a Lagrange-Remap anti-diffusive strategy following $[11,12]$. The numerical we propose here is conservative with respect to global mass, total energy momentum and partial masses. The approximate transport of the color functions follows the recursive construction of [11]: the update of $\mathcal{Z}_{k}$ has built-in stability and consistency, while ensuring that the summation of the color functions over all the materials equals to one.

The outline of the paper is as follows. We first introduce our $m$-component flow model. Then we present the Lagrange-Remap algorithm developed for discretizing this system in the case $m \geq 2$. Finally, we present a simulation of instability involving a triple point problem [9].

\section{A $m$-Component Extension of the Two-Component Five-Equation Model}

We consider a medium composed of $m \geq 2$ compressible materials. Each component is provided with an EOS $\left(\rho_{k}, p_{k}\right) \mapsto e_{k}\left(\rho_{k}, p_{k}\right)$, where $\rho_{k}, p_{k}, e_{k}$ are the density, partial pressure and specific interal energy of the fluid $k$. We recall that $\mathcal{Z}_{k}$ denotes the color function associated with the material $k$ : $\mathcal{Z}_{k}$ takes the value 1 in fluid $k$ and 0 elsewhere. The components share the same velocity $\mathbf{u}$. The density $\rho$ and the specific internal energy of the $m$-component medium are defined by

$$
\rho=\sum_{k=1}^{m} \mathcal{Z}_{k} \rho_{k}, \quad \rho e=\sum_{k=1}^{m} \mathcal{Z}_{k} \rho_{k} e_{k} .
$$

We note $E=e+|\mathbf{u}|^{2} / 2$ the specific total energy of the medium. The colour function $\mathcal{Z}_{k}$ is imposed to verify an immiscibility-type property that reads

$$
\sum_{k=1}^{m} \mathcal{Z}_{k}=1
$$

We consider the following generalization of the two-component five-equation model $[2,3]$

$$
\left\{\begin{array}{cl}
\partial_{t}(\rho \mathbf{u})+\nabla \cdot(\rho \mathbf{u} \otimes \mathbf{u})+\nabla p & =0 \\
\partial_{t}(\rho E)+\nabla \cdot(\mathbf{u}(\rho E+p)) & =0 \\
\partial_{t}\left(\rho_{k} \mathcal{Z}_{k}\right)+\nabla \cdot\left(\rho_{k} \mathcal{Z}_{k} \mathbf{u}\right) & =0, \quad k=1, \ldots, m \\
\partial_{t} \mathcal{Z}_{k}+\mathbf{u} \cdot \nabla \mathcal{Z}_{k} & =0, \quad k=1, \ldots, m
\end{array}\right.
$$

where the pressure $p$ is defined by the closure relation

$$
\rho e=\sum_{k=1}^{m} \mathcal{Z}_{k} \rho_{k} e_{k}\left(\rho_{k}, p\right)
$$

Let us note $\xi_{k}=\left(\partial \rho_{k} e_{k} / \partial p_{k}\right)_{\rho_{k}}$ and $c_{k}=\sqrt{\left(\partial p_{k} / \partial \rho_{k}\right) e_{k}+\left(p_{k} / \rho_{k}^{2}\right)\left(\partial p_{k} / \partial e_{k}\right)_{\rho_{k}}}$ the sound velocity in pure component $k$. For medium composed by $m$ Mie-Grüneisen materials existence and uniqueness of $p$ is granted without additional hypotheses. In particular, if each component $k$ is governed by a Perfect Gas EOS $p_{k}=$ $\left(\gamma_{k}-1\right) \rho_{k} e_{k}, k=1, \ldots, m$, then the medium pressure $p$ is defined by the following analytical formula

$$
p=(\gamma-1) \rho e, \quad \text { with } \frac{1}{\gamma-1}=\sum_{k=1}^{m} \frac{\mathcal{Z}_{k}}{\gamma_{k}-1} .
$$


Following the same lines as in $[2,3]$ one can state the following proposition, concerning the eigenstructure and the well-posedness of system (2).

Proposition 1. Suppose $\xi_{k}>0$, then system (2) with isobaric closure (3) is hyperbolic. It possesses $2 m+2$ eigenvalues $\{u-c, u, \ldots, u, u+c\}$ where $c$ is the global sound speed given by

$$
\rho c^{2}=\frac{1}{\xi} \sum_{k=1}^{m} \rho_{k} \mathcal{Z}_{k} \xi_{k} c_{k}^{2} \quad \text { with } \xi=\sum_{k=1}^{k} \mathcal{Z}_{k} \xi_{k}
$$

The field associated with the eigenvalues $u \pm c$ are genuinely non-linear and the fields associated with the multiple eigenvalue $u$ are linearly degenerate.

Remark 1. The initial multi-material interface problem now boils down to solving a global system of equations for a compressible flow with a mass balance equation and a passive scalar transport equation for each component.

\section{Anti-Diffusive Lagrange-Remap Scheme}

In this section, we present an anti-diffusive Lagrange-Remap method [8,10] for our $m$-component system (2) adapted from the $m=2$ case [4,12]. This discretization relies on a two-step splitting that decouples the acoustic effects taken into account by Lagrange step from the transport that is approximated within the Remap step. The Lagrange and Remap steps are respectively associated with genuinely nonlinear fields and linearly degenerate fields.

We present our method in the one-dimensional case. Multi-dimensional problems are treated by means of a dimensional splitting as in [12]. Let $\Delta t$ and $\Delta x$ be respectively the time and space step. The real line is discretized over the mesh $([i \Delta x,(i+1) \Delta x])_{i \in \mathbb{Z}}$. Let $A$ be any variable. We consider a single time-step from $t^{n}$ to $t^{n+1}$ and for the sake of readability a cell-centered value of $A$ evaluated at instant $t^{n}$, evaluated after the

Lagrange step and after the Projection step will be noted respectively $A_{i}, \widetilde{A}_{i}$ and $\widehat{A}_{i}$. We use the same notation for face-centered values with a $i+1 / 2$ subscript. We also note $\llbracket A \rrbracket_{i}=A_{i+1 / 2}-A_{i-1 / 2}$

\subsection{Lagrange step}

The Lagrangian step consists in solving the Euler equations in Lagrangian coordinates [8, 10]. Following [12], we perform this task using the acoustic scheme [6]. If we note $\lambda=\Delta t / \Delta x$ and $L_{i}=1+\lambda \llbracket u \rrbracket_{i}$, this reads

$$
L_{i}(\widetilde{\rho u})_{i}=(\rho u)_{i}-\lambda \llbracket p \rrbracket_{i}, \quad L_{i}(\widetilde{\rho e})_{i}=(\rho e)_{i}-\lambda \llbracket p u \rrbracket_{i}, \quad L_{i}\left(\widetilde{\rho_{k} \mathcal{Z}_{k}}\right)_{i}=\left(\rho_{k} \mathcal{Z}_{k}\right)_{i}, \quad \widetilde{\mathcal{Z}_{k, i}}=\mathcal{Z}_{k, i}
$$

where

$$
p_{i+1 / 2}=\frac{p_{i}+p_{i+1}}{2}-\frac{1}{2}(\rho c)_{i+1 / 2}\left(u_{i+1}-u_{i}\right), \quad u_{i+1 / 2}=\frac{u_{i}+u_{i+1}}{2}-\frac{1}{2} \frac{1}{(\rho c)_{i+1 / 2}}\left(p_{i+1}-p_{i}\right)
$$

with $(\rho c)_{i+1 / 2}=\sqrt{\max \left(\left(\rho c^{2}\right)_{i},\left(\rho c^{2}\right)_{i+1}\right) \min \left(\rho_{i}, \rho_{i+1}\right)}$ and $\left(\rho c^{2}\right)_{i}$ computed thanks to $(5)$.

\subsection{Time Step Choice}

As in [12], for the sake of stability for both Lagrange and remap step, we shall assume in the sequel that the time step verifies the following Courant-Friedrichs-Lewy (CFL) condition

$$
\lambda \max _{i \in \mathbb{Z}}\left(\left|u_{i+1 / 2}\right|,(\rho c)_{i+1 / 2} / \min \left(\rho_{i}, \rho_{i+1}\right)\right) \leq 1 .
$$




\subsection{Remap step}

The remap step accounts for the material transport of the fluid $[8,10]$. In this context we use the following general form (see [4])

$$
\left\{\begin{aligned}
(\widehat{\rho u})_{i} & =(\widetilde{\rho u})_{i}-\lambda \llbracket u \widetilde{\rho u} \rrbracket_{i}+\lambda \llbracket u \rrbracket_{i}(\widetilde{\rho u})_{i}, \\
(\widehat{\rho E})_{i} & =(\widetilde{\rho e})_{i}-\lambda \llbracket u \widetilde{\rho e} \rrbracket_{i}+\lambda \llbracket u \rrbracket_{i}(\widetilde{\rho e})_{i}, \\
\left(\widehat{\rho_{k} \mathcal{Z}_{k}}\right)_{i} & =\left(\widetilde{\rho_{k} \mathcal{Z}_{k}}\right)_{i}-\lambda \llbracket u \widetilde{\rho_{k} \mathcal{Z}_{k} \rrbracket_{i}}+\lambda \llbracket u \rrbracket_{i}\left(\widetilde{\rho_{k} \mathcal{Z}_{k}}\right)_{i}, \\
\widehat{\mathcal{Z}}_{k, i} & =\widetilde{\mathcal{Z}}_{k, i}-\lambda \llbracket u \widetilde{\mathcal{Z}_{k}} \rrbracket_{i}+\lambda \llbracket u \rrbracket_{i} \widetilde{\mathcal{Z}}_{k, i} .
\end{aligned}\right.
$$

While $u_{i+1 / 2}$ and $\llbracket u \rrbracket_{i}$ are defined in section 2.1, we shall detail in the sequel the construction of the Remap fluxes $\widetilde{\rho}_{i+1 / 2}, \widetilde{\rho u}_{i+1 / 2}, \widetilde{\rho e}_{i+1 / 2},\left(\widetilde{\rho_{k} \mathcal{Z}_{k}}\right)_{i+1 / 2}$ and $\widetilde{\mathcal{Z}_{k, i+1 / 2}}$.

\subsubsection{Anti-Diffusive Color Functions Remap Flux}

For each $k=1, \ldots, m$, we use the lines of $[5,6,12]$ and consider two real numbers $\omega_{k, i+1 / 2} \leq \Omega_{k, i+1 / 2}$ such that choosing $\widetilde{\mathcal{Z}_{k, i+1 / 2}} \in\left[\omega_{k, i+1 / 2}, \Omega_{k, i+1 / 2}\right]$ yields a consistent and stable discretization for $\mathcal{Z}_{k}$ under the assumption (7). Unfortunately, this selection process does not ensure that the unit sum constraint (1) will be verified by the approximation of $\mathcal{Z}_{k}, k=1, \ldots, m$. As proposed in [11], we adopt a recursive construction in order to fulfill this additional constraint. For $k=1$, we set

$$
d_{1, i+1 / 2}=\max \left(\omega_{1, i+1 / 2} ; 1-\sum_{l=2}^{m} \Omega_{l, i+1 / 2}\right), \quad D_{1, i+1 / 2}=\min \left(\Omega_{1, i+1 / 2} ; 1-\sum_{l=2}^{m} \omega_{l, i+1 / 2}\right),
$$

and choose $\widetilde{\mathcal{Z}}_{1, i+1 / 2} \in\left[d_{1, i+1 / 2}, D_{1, i+1 / 2}\right]$. Let $k=2, \ldots, m-1$, suppose that $\widetilde{\mathcal{Z}}_{l, i+1 / 2}$ are already known for $l \leq k$, then we define

$d_{k, i+1 / 2}=\max \left(\omega_{k, i+1 / 2} ; 1-\sum_{l=1}^{k-1} \tilde{\mathcal{Z}}_{l, i+1 / 2}-\sum_{l=k+1}^{m} \Omega_{l, i+1 / 2}\right), D_{k, i+1 / 2}=\min \left(\Omega_{k, i+1 / 2} ; 1-\sum_{l=1}^{k-1} \tilde{\mathcal{Z}}_{l, i+1 / 2}-\sum_{l=k+1}^{m} \omega_{l, i+1 / 2}\right)$

and choose $\widetilde{\mathcal{Z}}_{k, i+1 / 2} \in\left[d_{k, i+1 / 2}, D_{k, i+1 / 2}\right]$. For $k=m$, we use $\widetilde{\mathcal{Z}}_{m, i+1 / 2}=1-\sum_{l=1}^{m-1} \tilde{\mathcal{Z}}_{l, i+1 / 2}$.

Finally, the above procedure allows to define a set of intervals $\left[d_{k, i+1 / 2}, D_{k, i+1 / 2}\right], k=1, \ldots, m$ such that $\widetilde{\mathcal{Z}}_{k, i+1 / 2} \in\left[d_{k, i+1 / 2}, D_{k, i+1 / 2}\right]$ ensures that the numerical approximation of $\mathcal{Z}_{k}$ is stable, consistent and satisfies the unit sum constraint. Recall that the downwind value for the flux of $\widetilde{\mathcal{Z}}_{k}$ is $\widetilde{\mathcal{Z}}_{k, i+1 / 2}^{\text {do }}=\widetilde{\mathcal{Z}}_{k, i+1}$ if $u_{i+1 / 2}>0$ and $\widetilde{\mathcal{Z}}_{k, i+1 / 2}^{\text {do }}=\widetilde{\mathcal{Z}}_{k, i}$ otherwise. In order to limit the numerical diffusion of the color functions, we adopt the strategy proposed by $[5,7]$ and applied in $[4,11,12]$ : for $k=1, \ldots, m$ we choose $\widetilde{\mathcal{Z}}_{k, i+1 / 2}$ to be the closest value within $\left[d_{k, i+1 / 2}, D_{k, i+1 / 2}\right]$ to $\widetilde{\mathcal{Z}}_{k, i+1 / 2}^{\text {do }}$ the downwind value of $\mathcal{Z}_{k}$. This leads to set

$$
\widetilde{\mathcal{Z}}_{k, i+1 / 2}= \begin{cases}d_{k, i+1 / 2}, & \text { if } \widetilde{\mathcal{Z}}_{k, i+1 / 2}^{\text {do }}<d_{k, i+1 / 2} \\ \widetilde{\mathcal{Z}}_{k, i+1 / 2}^{\text {do }}, & \text { if } \widetilde{\mathcal{Z}}_{k, i+1 / 2}^{\text {do }} \in\left[d_{k, i+1 / 2}, D_{k, i+1 / 2}\right] \\ D_{k, i+1 / 2}, & \text { if } \widetilde{\mathcal{Z}}_{k, i+1 / 2}^{\text {do }}>D_{k, i+1 / 2}\end{cases}
$$

We define here the Remap fluxes for all the conservative quantities. We define the phasic density and specific internal energy in the whole computational domain by setting $\rho_{k}=\left(\rho_{k} \mathcal{Z}_{k}\right) / \mathcal{Z}_{k}, e_{k}=e_{k}\left(\rho_{k}, p\right)$ if $\mathcal{Z}_{k} \neq 0$ and 
$\rho_{k}=e_{k}=0$ otherwise. The quantites $\left(\widetilde{\rho}_{k},{\widetilde{\rho_{k}}}_{k}, \widetilde{u}\right)_{i+1 / 2}$ are set to the upwind value with respect to the velocity $u_{i+1 / 2}$, namely

$$
\left(\widetilde{\rho}_{k}, \widetilde{\rho_{k} e_{k}}, \widetilde{u}\right)_{i+1 / 2}= \begin{cases}\left(\widetilde{\rho}_{k}, \widetilde{\rho_{k}} e_{k}, \widetilde{u}\right)_{i}, & \text { if } u_{i+1 / 2}>0 \\ \left(\widetilde{\rho}_{k},{\widetilde{\rho_{k}} e_{k}}, \widetilde{u}\right)_{i+1}, & \text { if } u_{i+1 / 2} \leq 0\end{cases}
$$

Using the definition of the conservative variables and the color function fluxes defined in section 2.3.1, we obtain the following fluxes that complete the design of our numerical scheme:

$$
\begin{aligned}
\widetilde{\rho}_{i+1 / 2} & =\sum_{k=1}^{m} \widetilde{\mathcal{Z}}_{k, i+1 / 2} \widetilde{\rho}_{k, i+1 / 2}, & (\widetilde{\rho e})_{i+1 / 2} & =\sum_{k=1}^{m} \widetilde{\mathcal{Z}}_{k, i+1 / 2}\left(\widetilde{\rho_{k} e_{k}}\right)_{i+1 / 2}, \quad\left(\widetilde{\rho_{k} \mathcal{Z}_{k}}\right)_{i+1 / 2}=\widetilde{\mathcal{Z}}_{k, i+1 / 2} \widetilde{\rho}_{k, i+1 / 2} \\
\widetilde{\rho u}_{i+1 / 2} & =\widetilde{\rho}_{i+1 / 2} \widetilde{u}_{i+1 / 2}, & (\widetilde{\rho E})_{i+1 / 2} & =\widetilde{(\rho e)_{i+1 / 2}}+\left(\widetilde{u}_{i+1 / 2}\right)^{2} / 2 .
\end{aligned}
$$

\section{NumericAl RESUlt}

The numerical results presented in the sequel have been obtained thanks to hydrodynamic code Heracles [1] developed by E. Audit and his coworkers ${ }^{1}$ in which the main method of this paper has been implemented.

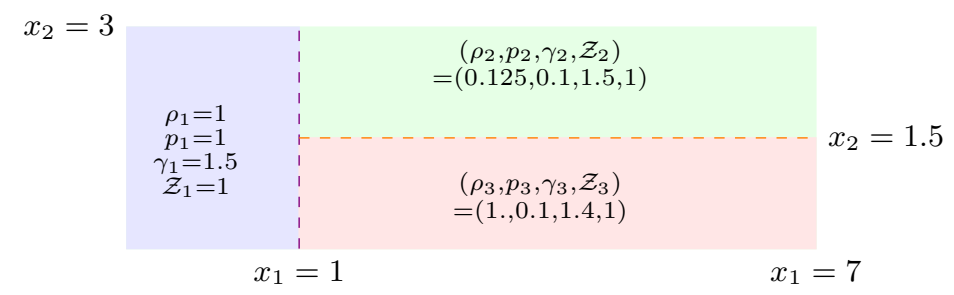

Figure 1: 2D triple point problem : geometry and initial data.

We proposed here a triple point test case dealing with a two-dimensional three-material Riemann problem in a rectangular domain whose dimensions and initial data are depicted on FIG. 1. This test has been examined in [9]. As we can see on FIG. 2 representing the interfaces at different times, a shock is propagating from the left material (blue) to the right two materials. As the green fluid is lighter then the two others, there is a Kelvin-Helmoltz like instability appearing. Globally, the numerical solution obtained is in good agreement with those found in the litterature (see [9]). In addition, one should observe the benefit of anti-diffusive scheme especially for the interface which remains sharp and then is precisely captured.

\section{Conclusion \& Future WOrK}

In this work, we have proposed an approach for the simulation of multi-materials flows with an arbitrary number of component using an extension of the andi-diffusive strategy based on the techniques presented in [11,12]. The numerical results are in good agreement with those obtained in the litterature. In particular, they demonstrate the great advantage of the andi-diffusive scheme which is simple to implement and very efficient especially to accurately treat the interface. Extension to the second order in space using MUSCL reconstruction is in progress adapting [4], especially to improve the nonlinear waves resolution.

\footnotetext{
${ }^{1}$ http://irfu.cea.fr/Projets/Site_heracles/index.html
} 

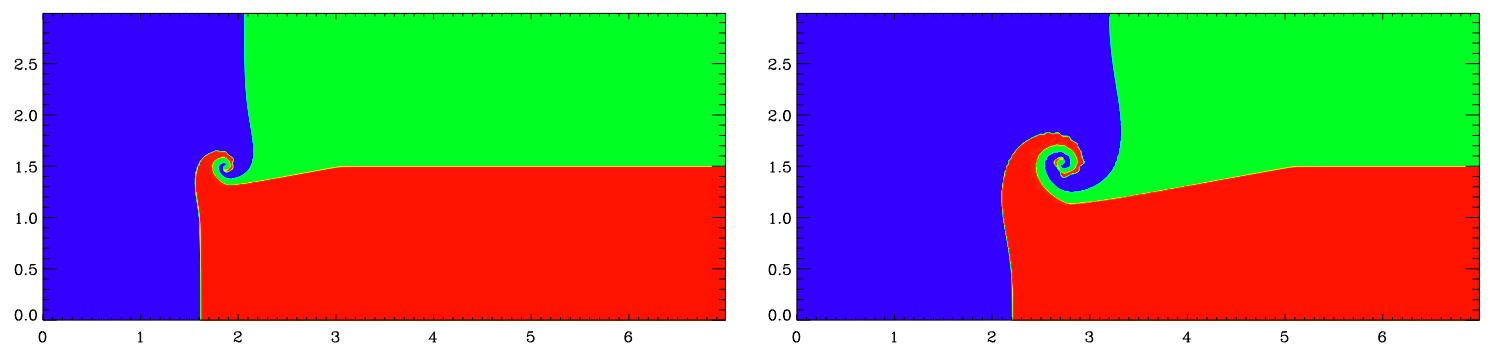

$t=1.2$
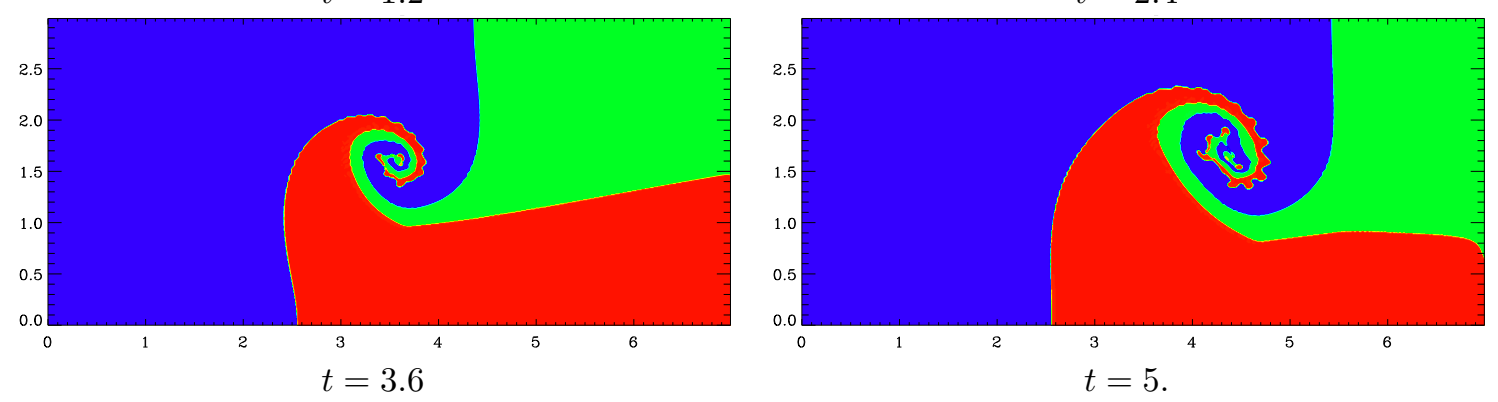

Figure 2: Triple point test case: mapping of the color function for $t \in\{1.2,2.4,3.6,5\}$ with the anti-diffusive scheme (bottom) for a $700 \times 300$ element mesh.

\section{Acknowledgments}

The presented work was partially supported by project SINERGY (Simulation Numérique pour la Recherche en Rayonnement, Gravitation et Hydrodynamique, ANR 06-C86-009) of the french ANR (Agence Nationale de la Recherche) .

\section{REFERENCES}

[1] E. Audit, M. Gonzalez, P. Huynh: Heracles: a three dimensional radiation hydrodynamics code, Astronomy and Astrophysics, 2007, volume 464, issue 2, March 2007, pp. 429-435.

[2] G. Allaire, S. Clerc, S. Kokh: A five-equation model for the numerical simulation of interfaces in two-phase flows, C. R. Acad. Sci. Paris, Série I, t. 331, 2000, pp. 1017-1022.

[3] G. Allaire, S. Clerc and S. Kokh: A Five-Equation Model for the Simulation of Interfaces between Compressible Fluids, Journal of Computational Physics, Volume 181, Issue 2, 20 September 2002, pp. 577-616.

[4] M. Billaud Friess, B. Boutin, F. Caetano, G. Faccanoni, S. Kokh, F. Lagoutière and L. Navoret: A second order antidiffusive Lagrange-remap scheme for two-component flows, ESAIM: PROCEEDINGS, October 2011, Vol. 32, p. $149-162$.

[5] B. Després, F. Lagoutière: Un schéma non linéaire anti-dissipatif pour l'équation d'advection linèaire, Comptes Rendus de l'Acadèemie des Sciences - Series I - Mathematics, Volume 328, Issue 10, 15 May 1999, pp. 939-943.

[6] B. Després: Inégalite entropique pour un solveur conservatif du système de la dynamique des gaz en coordonnées de Lagrange, Comptes Rendus de l'Acadèmie des Sciences - Series I - Mathematics, Volume 324, Issue 11, June 1997, pp. $1301-1306$.

[7] B. Després, F. Lagoutière: Numerical resolution of a two-component compressible fluid model with interfaces, Progress in Computational Fluid Dynamics 7, no 6, 2007, pp. 295-310.

[8] B. Després: Lois de Conservations Eulériennes, Lagrangiennes et Méthodes Numériques, Mathématiques et applications, SMAI, Springer, volume 68, 2010.

[9] S. Galéra, J. Breil and P.-H. Maire: A 2D unstructured multi-material Cell-Centered Arbitrary Lagrangian-Eulerian (CCALE) scheme using MOF interface reconstruction, Computers \& Fluids, Volume 46, Issue 1, 10th ICFD Conference Series on Numerical Methods for Fluid Dynamics (ICFD 2010), July 2011, pp. 237-244.

[10] E. Godlewski, P.-A. Raviart: Numerical approximation of hyperbolic systems of conservation laws, Springer, 1996.

[11] S. Jaouen and F. Lagoutière: Numerical transport of an arbitrary number of components, Computer Methods in Applied Mechanics and Engineering, Volume 196, Issues 33-34, 1 July 2007, pp. 3127-3140. 
[12] S. Kokh, F. Lagoutière: An anti-diffusive numerical scheme for the simulation of interfaces between compressible fluids by means of a five-equation model, Journal of Computational Physics, Volume 229, Issue 8, 20 April 2010, pp. $2773-2809$. 